Bull. Austral. Math. Soc.

VoL. 52 (1995) [341-344]

\title{
AN EXTENSION OF THE GENERALISED SCHUR INEQUALITY
}

\author{
B. MOND AND J.E. PeČArić
}

The well-known Schur inequality relates the sum of the squares of the absolute values of the eigenvalues of $A$ to the elements of $A$. This was recently generalised to powers between one and two. Here we show that the inequality holds for powers between zero and two.

Let $A$ be an $n \times n$ matrix, real or complex, with eigenvalues $\lambda_{1}, \ldots, \lambda_{n}$. The Schur inequality

$$
\sum_{i=1}^{n}\left|\lambda_{i}\right|^{2} \leqslant \sum_{i, j=1}^{n}\left|a_{i j}\right|^{2}
$$

is well-known $[2, \mathrm{p} .133]$.

Petri and Ikramov [3] generalised the Schur inequality to

$$
\sum_{i=1}^{n}\left|\lambda_{i}\right|^{p} \leqslant \sum_{i, j=1}^{n}\left|a_{i j}\right|^{p}
$$

where $1 \leqslant p \leqslant 2$.

Ikramov [1] proved, for any $n \times n$ matrix $A$ with singular values $s_{1}, s_{2}, \ldots, s_{n}$, the following result:

$$
\sum_{i=1}^{n} s_{i}^{p} \leqslant \sum_{i, j=1}^{n}\left|a_{i j}\right|^{p}
$$

where $1 \leqslant p \leqslant 2$.

Now (2) is a simple consequence of (3) by the well-known Weyl inequality:

We assume that the singular values of $A$ constitute a non-increasing sequence

$$
s_{1} \geqslant s_{2} \geqslant \ldots \geqslant s_{n}
$$

Received 16th January, 1995

Copyright Clearance Centre, Inc. Serial-fee code: 0004-9729/95 \$A2.00+0.00. 
and that the eigenvalues of $A$ are numbered in accordance with their magnitudes

$$
\left|\lambda_{1}\right| \geqslant\left|\lambda_{2}\right| \geqslant \ldots \geqslant\left|\lambda_{n}\right| \text {. }
$$

Then for $1 \leqslant k \leqslant n$ and $0<p<\infty$

$$
\sum_{i=1}^{k}\left|\lambda_{i}\right|^{p} \leqslant \sum_{i=1}^{k} s_{i}^{p} .
$$

We now have

TheOREM 1. For any $n \times n$ matrix $A$ with singular values $s_{1}, \ldots, s_{n}$, inequality (3) is valid for $0<p \leqslant 2$. For $p \geqslant 2$, the reverse inequality holds.

Proof: The proof follows closely that of Theorem 1 of $[1]$. Let $\tau_{1}, \ldots, \tau_{n}$ denote the $\ell_{2}$ norms of the row vectors $\left(a_{i 1}, a_{i 2}, \ldots, a_{i n}\right)$ numbered so that they form the non-increasing sequence

$$
\tau_{1} \geqslant \tau_{2} \geqslant \ldots \geqslant \tau_{n}
$$

It is well-known [3] that the sequence $\tau_{1}^{2}, \ldots, \tau_{n}^{2}$ is majorised by $s_{1}^{2}, \ldots, s_{n}^{2}$, that is,

$$
\begin{gathered}
\sum_{i=1}^{k} \tau_{i}^{2} \leqslant \sum_{i=1}^{k} s_{i}^{2}, \quad 1 \leqslant k \leqslant n \\
\sum_{i=1}^{n} \tau_{i}^{2}=\sum_{i=1}^{n} s_{i}^{2} .
\end{gathered}
$$

So if $f$ is a concave function, we have

$$
\sum_{i=1}^{n} f\left(s_{i}^{2}\right) \leqslant \sum_{i=1}^{n} f\left(\tau_{i}^{2}\right)
$$

and the reverse inequality holds in (5) if $f$ is a convex function.

In particular, the function $f(x)=x^{p / 2}$ is concave for $x>0$ if $0<p \leqslant 2$ and convex for $p \geqslant 2$. Therefore

$$
\sum_{i=1}^{n} s_{i}^{p} \leqslant \sum_{i=1}^{n} \tau_{i}^{p}
$$

holds for $0<p \leqslant 2$ and the reverse inequality holds for $p \geqslant 2$.

On the other hand, for $\ell_{p}$ norms of any row vector, we have

$$
\left(\sum_{j=1}^{n}\left|a_{i j}\right|^{2}\right)^{1 / 2} \leqslant\left(\sum_{j=1}^{n}\left|a_{i j}\right|^{p}\right)^{1 / p}
$$


for $0<p \leqslant 2$ and the reverse inequality for $p \geqslant 2$.

Taking the $p$ th power of both sides in (7) and adding the inequalities for $i=$ $1, \ldots, n$, we obtain

$$
\sum_{i=1}^{n} \tau_{i}^{p} \leqslant \sum_{i, j=1}^{n}\left|a_{i j}\right|^{p}
$$

for $0<p \leqslant 2$, and the reverse inequality for $p \geqslant 2$.

The assertion of the theorem now follows from (6) and (8) and their reversals.

Theorem 2. Let $A$ be an $n \times n$ matrix, real or complex, with eigenvalues $\lambda_{1}, \ldots, \lambda_{n}$. Then (2) is valid for $0<p \leqslant 2$.

Proof: This is a simple consequence of Theorem 1 and Weyl's inequalities (4).

\section{REFERENCES}

[1] K.D. Ikramov, 'A simple proof of the generalized Schur inequality', Linear Algebra Appl. 189 (1994), 143-149.

[2] L. Mirsky, An introduction to linear algebra (Oxford University Press, Oxford, 1955).

[3] N.V. Petri and K.D. Ikramov, 'Extremal properties of some matrix norms', U.S.S.R. Comput. Math. and Math. Phys. 8 No. 4 (1968), 219-230.

Department of Mathematics

La Trobe University

Bundoora Vic 3083

Australia
Faculty of Textile Technology

University of Zagreb

Zagreb

Croatia

and

Department of Mathematics

La Trobe University

Bundoora Vic $\mathbf{3 0 8 3}$

Australia 